\title{
The impact of intravenous dexamethasone on the efficacy and duration of analgesia of paravertebral block in breast cancer surgery: a randomized controlled trial
}

This article was published in the following Dove Press journal:

Journal of Pain Research

\author{
Ahmed H Bakeer' \\ Nasr M Abdallah ${ }^{2}$ \\ Mahmoud A Kamel' \\ Dina N Abbas' \\ Ahmed S Ragab' \\ 'Department of Anesthesia and \\ Pain Management, National Cancer \\ Institute, Cairo University, Giza, Egypt; \\ ${ }^{2}$ Department of Anesthesia, Faculty of \\ Medicine, Cairo University, Giza, Egypt
}

Correspondence: Ahmed H Bakeer Department of Anesthesia and Pain Management, National Cancer Institute, Cairo University, 13 Mohamed Shokry Street, Agouza I24II, Giza, Egypt $\mathrm{Tel}+20233360906$

Email mail@mcs-center.com
Purpose: The study aimed at the evaluation of the impact of intravenous (IV) dexamethasone on efficacy and duration of analgesia of paravertebral block (PVB) in patients undergoing modified radical mastectomy (MRM).

Patients and methods: This randomized, double-blind controlled trial included 50 patients with breast cancer scheduled for unilateral MRM. Ultrasound-guided PVB was performed in outof-plane technique. The technique was repeated at each segment from C7 to T6. The participants were randomly allocated to one of two groups. Group BD ( $\mathrm{n}=25)$ received IV $8 \mathrm{mg}$ dexamethasone diluted with $8 \mathrm{~mL}$ of normal saline to reach $10 \mathrm{~mL}$ solution, while Group B received IV $10 \mathrm{~mL}$ normal saline. Top-up local infiltration analgesia into the surgical field was performed by the surgeon if needed using lidocaine $1 \%$ intraoperatively. Propofol infusion of $50-100 \mu \mathrm{g} / \mathrm{kg} / \mathrm{min}$ was maintained throughout the surgery. The time to administration of the first postoperative analgesic dose, pain intensity as visual analog scale (VAS) score, number of patients who required rescue morphine analgesia, total morphine consumption, postoperative nausea and vomiting (PONV) impact scale, and the overall satisfaction of patients with pain management were measured.

Results: Fifty patients were randomized and analyzed. The time to first rescue analgesic dose was significantly longer in Group DB $(P<0.001)$. The VAS scores were significantly lower in Group DB compared to Group B up to 12 hours postoperatively. Morphine consumption was lower in Group DB compared to Group B. PONV Impact Scale score was significantly higher in Group B. Conclusion: Systemic dexamethasone increased the efficacy and duration of the single-shot multilevel PVB in breast cancer surgery.

Trial registration: ISRCTN registry, study ID: ISRCTN15920148

Keywords: breast cancer, paravertebral block, dexamethasone, postoperative pain, regional anesthesia

\section{Introduction}

Breast cancer is the most frequent malignant neoplasm in females. ${ }^{1}$ Surgical procedures for the management of breast cancer are typically performed under general anesthesia. Breast cancer surgery is not always associated with serious complications, and early recovery is always achieved, making them ideal candidates for ambulatory surgery. ${ }^{2}$ However, postoperative pain, nausea, and vomiting are commonly encountered after breast surgery which may hinder early recovery. ${ }^{3}$

Regional anesthesia using paravertebral block (PVB) has been suggested as a good alternative to general anesthesia for breast cancer surgery. It provides better 
postoperative analgesia with reduction of the risk of nausea, vomiting, and chronic pain. ${ }^{4}$ PVB exerts its analgesic effect via elimination of cortical responses to thoracic dermatomal stimulation. ${ }^{5}$ Moreover, PVB has been suggested to reduce the risk of recurrence or metastasis in patients undergoing modified radical mastectomy (MRM). ${ }^{6}$

Nevertheless, the postoperative analgesic duration is not adequate to compensate for the acute nociception associated with surgery in the early perioperative phase of healing. Many adjuvants including clonidine, neostigmine, epinephrine, tramadol, buprenorphine, and dexamethasone have been added to local anesthetics (LAs) to enhance postoperative analgesia. $^{7}$ In 2006, Movafegh et $\mathrm{al}^{8}$ found that dexamethasone has improved the action of lidocaine as regards the duration of axillary brachial plexus blockade.

Dexamethasone is the most potent anti-inflammatory drug with a long half-life. ${ }^{9}$ A single dose of $8 \mathrm{mg}$ of dexamethasone has been found to produce effective postoperative analgesia following dental surgery ${ }^{10}$ and thyroidectomy for up to 48 hours. ${ }^{11}$ A recent meta-analysis showed that an intermediate intravenous (IV) dose of $0.11-0.2 \mathrm{mg} / \mathrm{kg}$ has an opioid-sparing effect. ${ }^{12}$ Moreover, in 2013, it has been suggested that IV route can produce a comparable effect of perineural dexamethasone. ${ }^{13}$

Postoperative nausea and vomiting (PONV) is a common complication of all operative procedures. Breast cancer surgery under general anesthesia is associated with a relatively high incidence of PONV; it affects $60 \%-80 \%$ of patients undergoing mastectomy during the first 24 hours. ${ }^{14}$ PONV prolongs recovery and may lead to aspiration of gastric contents, wound dehiscence, and psychological distress. ${ }^{15}$

The aim of this study was to evaluate the effect of a single IV dose of dexamethasone on the efficacy and duration of analgesia of PVB in patients undergoing MRM.

\section{Patients and methods}

The study was conducted at the National Cancer Institute (NCI), Cairo University, Egypt, in the Department of Surgery during the period from June 2017 to October 2017. The study implemented the principles of the Declaration of Helsinki (1964) and its following revisions. The Institutional Review Board approved the study (approval no. $201617016.2 \mathrm{p}$, on 18 June 2017) and registered on the ISRCTN registry with study ID ISRCTN15920148. All data regarding the technical benefits, expectations, and hazards were explained to all patients and written informed consent was obtained from all patients. The patients were eligible if they were at least
18 years old, with breast cancer, scheduled for MRM, and able to understand and willing to follow the study protocol.

Exclusion criteria included patients with contraindications to PVB (coagulopathies, severe bronchopulmonary disease, prior contralateral pneumonectomy, and preexisting neuropathy involving the surgical site), failed PVB, patient refusing regional anesthesia, allergy or intolerance to one or more medications of the study protocol, chronic pain syndrome, chronic opioid use, and chronic systemic corticosteroid use.

\section{Intervention}

In the preoperative area, IV access was established on the contralateral side of the surgical procedure. IV dexmedetomidine $0.5-1.0 \mu \mathrm{g} / \mathrm{kg}$ and fentanyl $0.5-1.0 \mu \mathrm{g} / \mathrm{kg}$ were administered. Noninvasive blood pressure, pulse oximetry, and electrocardiogram monitoring were established. With the patient lying in the lateral position with the side of block up, sterilization with povidone-iodine, draping, and skin infiltration with lidocaine 1\% (1 $\mathrm{mL}$ ) was done. Then, PVB was performed with transportable ultrasound equipment with a $50 \mathrm{~mm}$ linear $15-6 \mathrm{MHz}$ probe (SonoSite M-Turbo ${ }^{\mathrm{TM}}$, SonoSite Inc., Bothell, WA, USA). An out-of-plane needle guidance technique with the needle positioned $1 \mathrm{~cm}$ caudal to the ultrasound probe was performed. A 22-G spinal needle was used. We used the hydrolocation technique by injecting $0.5-1 \mathrm{~mL}$ saline to localize the needle tip and confirm proper placement in the paravertebral space by observing anterior displacement of the parietal pleural upon injection. Once the tip of the needle was in its proper position, bupivacaine $0.5 \%$ with epinephrine $1 / 200,000,3-4 \mathrm{~mL}$ was injected. The technique was repeated at each segment from C7 to T6 (Figure 1). The success of the block was assessed by temperature sensation loss over the required dermatomes and by testing pinprick sensation before surgical incision.

\section{Randomization, allocation, and masking of study groups}

After assessment of the block, patients' randomization was done by a clinical nurse independent of the protocol who obtained a randomization number (computer-generated random numbers), with proper concealment in opaque sealed envelopes. The patient was then allocated to Group B or Group BD. To maintain blinding, the anesthesiologist who evaluated the patient could not guess allocation at any time.

Group BD $(\mathrm{n}=25)$ received IV $8 \mathrm{mg}$ dexamethasone diluted with $8 \mathrm{~mL}$ normal saline to reach $10 \mathrm{~mL}$ solution, while Group B ( $\mathrm{n}=25)$ received IV $10 \mathrm{~mL}$ normal saline. 


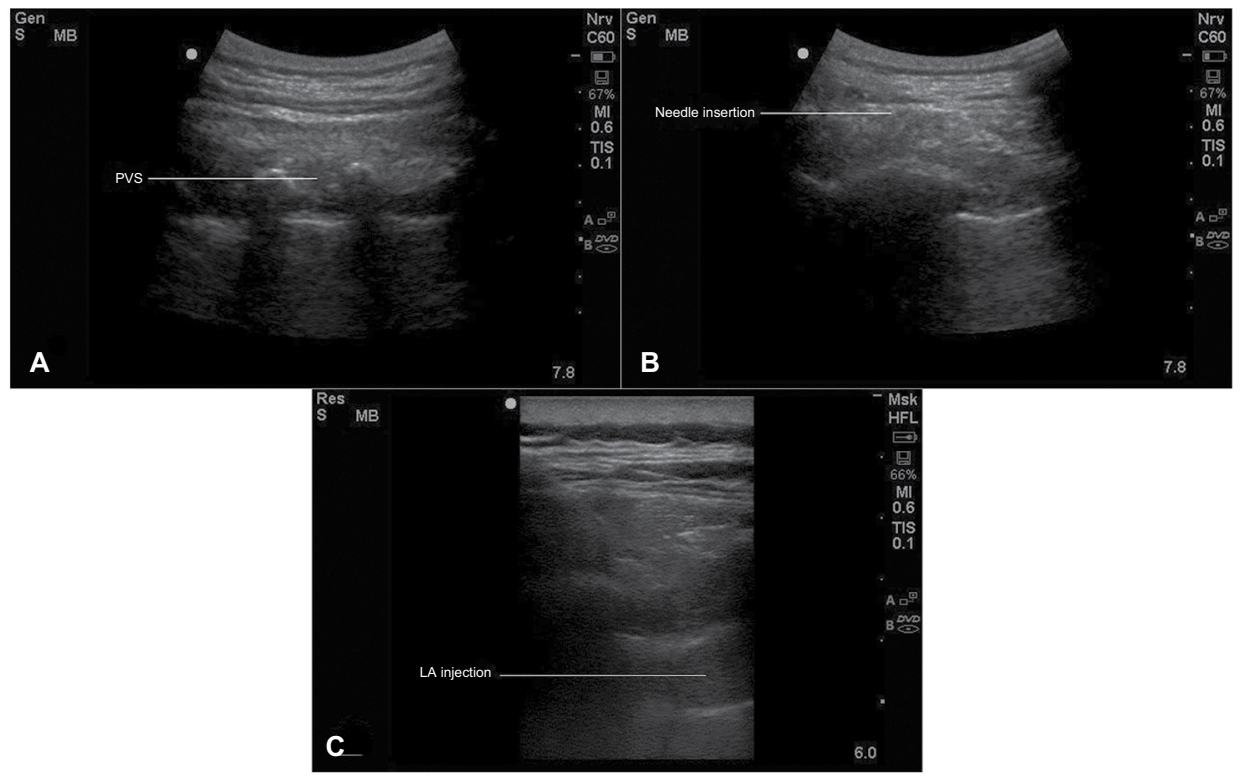

Figure I Technique of paravertebral block (A) Localization of PVS; (B) Needle insertion into the PVS; (C) Injection of LA. Abbreviations: LA, local anesthetic; PVS, paravertebral space.

Top-up local infiltration analgesia into the surgical field was performed by the surgeon, if needed for any patient, in the two groups using lidocaine $1 \%$ intraoperatively.

Patients were evaluated for 24 hours for pain intensity and duration, rescue analgesics, and drug adverse effects. Pain intensity was assessed on a visual analog scale (VAS) from 0 to $10(0=$ no pain at all to $10=$ worst pain imaginable $)$. If the VAS score was $\geq 4$, a $3 \mathrm{mg}$ dose of morphine was administered every 4 hours as needed. PONV was evaluated according to the PONV Impact Scale ${ }^{16}$ with scores 0-6. A score of $\geq 5$ defines clinically important PONV. The overall satisfaction of patients with pain management given to them in the postoperative phase was assessed on a scale of five categories (very satisfied, satisfied, slightly satisfied, slightly dissatisfied, and very dissatisfied). ${ }^{17}$

The primary outcome measure was the duration of analgesia defined as the time till the first administration of analgesic postoperatively. The secondary outcome measures were pain intensity, the number of patients who required rescue morphine analgesia, total morphine consumption, and PONV.

\section{Sample size estimation}

No previous studies tested the effect of adding IV dexamethasone to augment the analgesic effect of PVB. Thus, we calculated the required sample size based on a study using the same technique for interscalene block. According to this study, ${ }^{18}$ based on the primary outcome measure (duration of analgesia), seven patients were required to have a $95 \%$ chance of detecting, as significant at the $5 \%$ level, a longer analgesic duration in the experimental group (19.1 \pm 5.2 hours) compared to $11.8 \pm 2.4$ hours in the control group. Given the difference in technique, disease type, and surgical procedure, we included 25 patients in each group to test the effect of adding IV dexamethasone on pain control, opioid consumption, and hemodynamic stability.

\section{Statistical analyses}

Statistical analyses were done using $\mathrm{IBM}^{\odot}$ SPSS $^{\odot}$ Statistics version 22 (IBM Corporation, Armonk, NY, USA). The sample size was estimated using the online power calculator for continuous outcome superiority trial under Sealed Envelope Ltd., 2012 (available from https://www.sealedenvelope. com/power/continuous-superiority/). Numerical data were expressed as mean and SD or median and range as appropriate. Qualitative data were expressed as frequency and percentage. Chi-squared test (Fisher's exact test) was used to examine the relationship between qualitative variables. For quantitative data, the comparison between two groups was made using independent sample $t$-test or Mann-Whitney $U$-test as appropriate. Comparison of repeated measures was done using Friedman test. $P$-values were corrected for multiple analyses. A $P$-value $<0.05$ was considered significant.

\section{Results}

Fifty patients were randomized and analyzed (Figure 2). There was no significant difference between the two groups 
regarding baseline demographic and clinical characteristics (Table 1).

Duration of analgesia dose was significantly longer in Group DB $(P<0.001)$. Between 2 hours and postoperatively up to 12 hours, VAS scores were significantly lower in Group DB compared to Group B (Table 2). By the end of the observation period (at 24 hours), the two groups had comparable VAS score $(P=0.216)$. Ten $(40 \%)$ patients of Group DB did not request any further analgesic during the 24 postoperative hours compared to only one in Group B, while $56 \%$ of the latter needed two doses of morphine $(P<0.001)$. The total morphine consumption was significantly lower in Group DB compared to Group B $(P<0.001)$. PONV Impact Scale score was significantly higher in Group $\mathrm{B}(P=0.001)$. However, no patients had clinically important PONV (Table 2).

The mean arterial pressure was comparable in the two groups; all of the readings were within the clinically accepted range (Figure 3). The patients' rating of their satisfaction with postoperative pain management procedure is illustrated in Figure 4.

\section{Discussion}

The present study demonstrated that a preoperative IV dose of $8 \mathrm{mg}$ dexamethasone prolonged the analgesic duration of PVB, decreased pain intensity during the first 12 postoperative hours, and reduced opioid consumption in patients undergoing MRM for breast cancer under multilevel PVB. Dexamethasone was also associated with lower PONV Impact Scale score.

Regional anesthesia was adopted by many authorities and was found to enhance analgesia, and reduce perioperative morbidity, with consequently shorter hospital stay. ${ }^{19-21}$ Of the regional techniques, PVB has been shown to be effective in women having major breast surgeries..$^{22,23}$

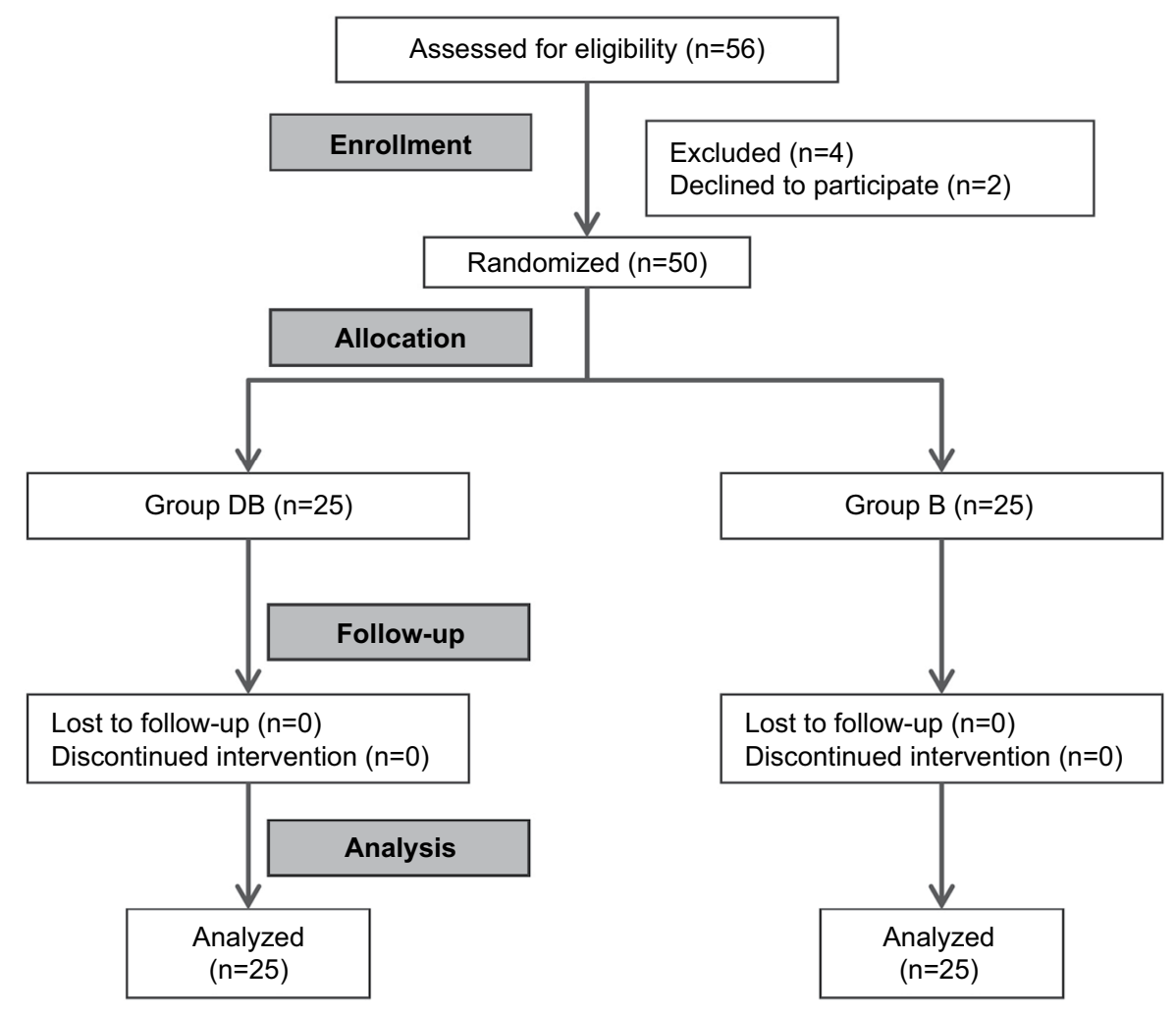

Figure 2 CONSORT flowchart.

Table I Baseline demographic and clinical characteristics of the two studied groups

\begin{tabular}{|l|l|l|l|}
\hline Baseline characteristics & Group DB $(\mathbf{n}=\mathbf{2 5})$ & Group B $(\mathbf{n}=\mathbf{2 5})$ & P-value \\
\hline Age (years) & $48.3 \pm 8.5$ & $49.8 \pm 8.7$ & 0.524 \\
\hline Weight $(\mathrm{kg})$ & $69.7 \pm 6.5$ & $71.6 \pm 3.9$ & 0.065 \\
\hline Mean arterial pressure $(\mathrm{mmHg})$ & $81 \pm 8$ & $83 \pm 9$ & 0.410 \\
\hline
\end{tabular}

Note: Data are presented as mean \pm SD. 
Table 2 Analgesic profile during the postoperative 24 hours of the two studied groups

\begin{tabular}{|c|c|c|c|}
\hline & Group DB $(n=25)$ & Group B $(n=25)$ & $P$-value \\
\hline Time to first analgesic dose (minutes) & $224 \pm 30$ & $173 \pm 24$ & $<0.001$ \\
\hline \multicolumn{4}{|l|}{ VAS } \\
\hline At 2 hours & $0(0-3)$ & $4(2-6)$ & $<0.001$ \\
\hline At 6 hours & $3(I-4)$ & $5(2-7)$ & $<0.001$ \\
\hline At 12 hours & $3(2-4)$ & $5(2-6)$ & $<0.001$ \\
\hline At 24 hours & $3(2-5)$ & $3(I-5)$ & 0.216 \\
\hline \multicolumn{4}{|c|}{ Number of patients requiring additional morphine doses } \\
\hline Single dose & $13(52.0 \%)$ & $10(40.0 \%)$ & \multirow[t]{2}{*}{$<0.001$} \\
\hline Two doses & $2(8.0 \%)$ & $14(56.0 \%)$ & \\
\hline 24 hours morphine dose $(\mathrm{mg})$ & $6.8 \pm 3.5$ & $10.5 \pm 4.2$ & \\
\hline PONV scale & $I(0-3)$ & $2(I-3)$ & 0.001 \\
\hline
\end{tabular}

Note: Data are presented as mean \pm SD, median (range), or $n(\%)$.

Abbreviation: PONV, postoperative nausea and vomiting; VAS, visual analog scale.

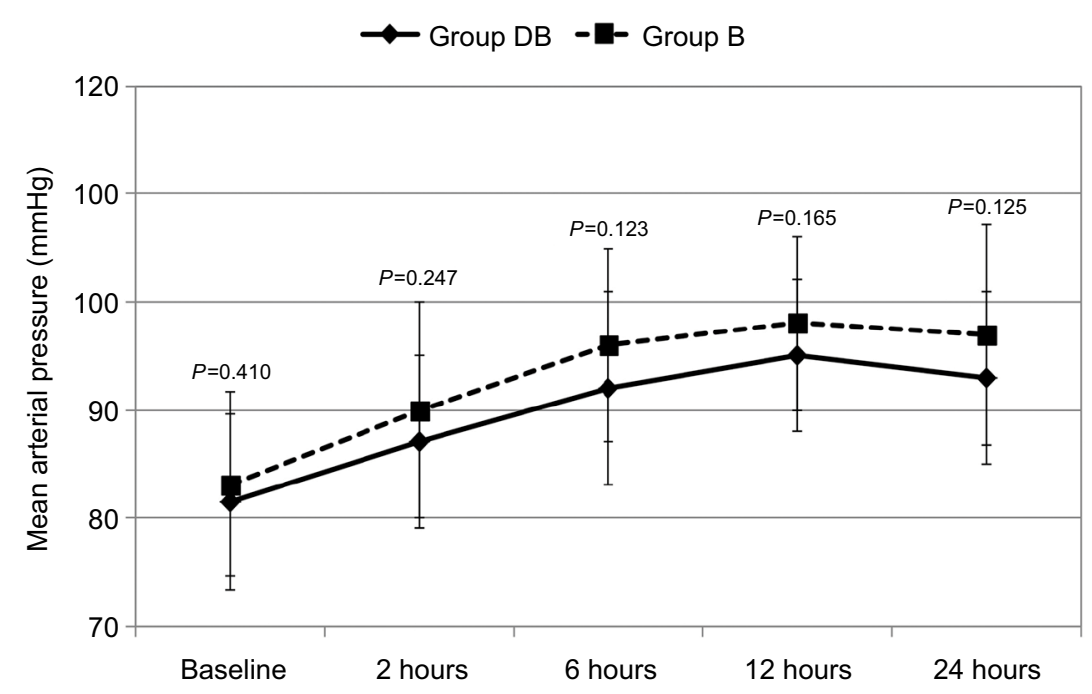

Figure 3 Changes of mean arterial pressure during the 24 postoperative hours.

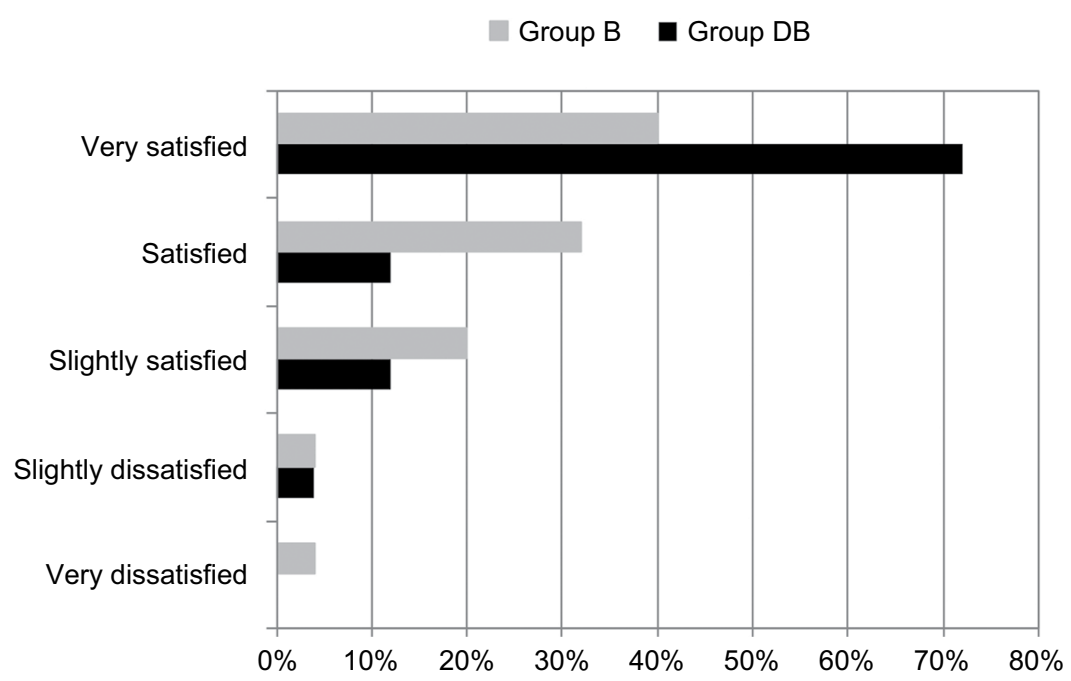

Figure 4 Bar graph representing the overall level of patient satisfaction. 
However, duration of postoperative analgesia constitutes a principal limitation of PVB in breast surgery. ${ }^{20,22}$ In this study, we used dexamethasone in a dose of $8 \mathrm{mg}$ preoperatively to prolong the analgesic duration of PVB. ${ }^{12}$ In this concern, Desmet et a ${ }^{13}$ found that IV and perineural dexamethasone are equivalent in increasing the analgesic duration of a singleshot interscalene block with ropivacaine for shoulder surgery.

Few studies are available about adding dexamethasone to PVB in breast cancer surgery. In a small case series of breast cancer surgeries, Goravanchi et $\mathrm{al}^{24}$ found that adding dexamethasone to the LA prolonged the duration of PVB. Additionally, IV dexamethasone was found to be valuable in postoperative pain control and reduction of opioid consumption when given in intermediate dose $(0.1-0.2 \mathrm{mg} / \mathrm{kg}){ }^{25} \mathrm{~A}$ systematic review of 24 randomized clinical trials, including 2,751 patients, reported that IV dexamethasone at doses more than $0.1 \mathrm{mg} / \mathrm{kg}$ is an effective adjunct in multimodal strategies in reducing postoperative pain and opioid consumption after surgery. This review included a heterogeneous group of procedures involving head and neck, abdominal, orthopedic, and gynecology surgeries. ${ }^{12}$ The exact mechanism of analgesic action of dexamethasone is not well-understood. However, it appears that it works systemically on decreasing cyclooxygenase and lipoxygenase production by inhibiting peripheral phospholipase. ${ }^{26,27}$ This study supports the concept of improving the postoperative analgesic efficacy of PVB by adding a single IV dose of dexamethasone.

The limitation of this study is that the perineural effect of dexamethasone was not studied and compared to the systemic efficacy. It is recommended that further randomized controlled studies, to identify which specific combinations of multimodal analgesia components, are most suitable for patients in each operative and postoperative setting.

\section{Conclusion}

This study shows that systemic dexamethasone increased the efficacy and duration of the single-shot multilevel PVB in breast surgery.

\section{Data sharing statement}

The authors declare that deidentified participants' data will be available whenever requested. The corresponding author will be ready to send the data for any authority on request by an e-mail message. The data will be available this way up to 3 months after being published.

\section{Disclosure}

The authors report no conflicts of interest in this work.

\section{References}

1. Ferlay J, Soerjomataram I, Dikshit R, et al. Cancer incidence and mortality worldwide: sources, methods and major patterns in GLOBOCAN 2012. Int J Cancer. 2015;136(5):E359-E386.

2. Ng YY, Chan PM, Chen JJ, Seah MD, Teo C, Tan EY. Adopting ambulatory breast cancer surgery as the standard of care in an Asian population. Int J Breast Cancer. 2014;2014:672743.

3. Coveney E, Weltz CR, Greengrass R, et al. Use of paravertebral block anesthesia in the surgical management of breast cancer: experience in 156 cases. Ann Surg. 1998;227(4):496-501.

4. Naccache N, Jabbour H, Nasser-Ayoub E, Abou Zeid H, Naja Z. Regional analgesia and breast cancer surgery. JMed Liban. 2009;57(2): 110-114.

5. Klein SM, Bergh A, Steele SM, Georgiade GS, Greengrass RA. Thoracic paravertebral block for breast surgery. Anesth Analg. 2000;90(6):1402-1405.

6. Exadaktylos AK, Buggy DJ, Moriarty DC, Mascha E, Sessler DI. Can anesthetic technique for primary breast cancer surgery affect recurrence or metastasis? Anesthesiology. 2006;105(4):660-664.

7. Knezevic NN, Anantamongkol U, Candido KD. Perineural dexamethasone added to local anesthesia for brachial plexus block improves pain but delays block onset and motor blockade recovery. Pain Physician. 2015;18(1):1-14.

8. Movafegh A, Razazian M, Hajimaohamadi F, Meysamie A. Dexamethasone added to lidocaine prolongs axillary brachial plexus blockade. Anesth Analg. 2006;102(1):263-267.

9. de Oliveira GS, Ahmad S, Fitzgerald PC, et al. Dose ranging study on the effect of preoperative dexamethasone on postoperative quality of recovery and opioid consumption after ambulatory gynaecological surgery. Br J Anaesth. 2011;107(3):362-371.

10. Numazaki M, Fujii Y. Reduction of postoperative emetic episodes and analgesic requirements with dexamethasone in patients scheduled for dental surgery. J Clin Anesth. 2005;17(3):182-186.

11. Worni M, Schudel HH, Seifert E, et al. Randomized controlled trial on single dose steroid before thyroidectomy for benign disease to improve postoperative nausea, pain, and vocal function. Ann Surg. 2008;248(6): 1060-1066.

12. de Oliveira GS, Almeida MD, Benzon HT, Mccarthy RJ. Perioperative single dose systemic dexamethasone for postoperative pain: a meta-analysis of randomized controlled trials. Anesthesiology. 2011;115(3):575-588.

13. Desmet M, Braems H, Reynvoet $M$, et al. IV and perineural dexamethasone are equivalent in increasing the analgesic duration of a single-shot interscalene block with ropivacaine for shoulder surgery: a prospective, randomized, placebo-controlled study. Br J Anaesth. 2013;111(3):445-452.

14. Reihnér E, Grunditz R, Giesecke K, Gustafsson LL. Postoperative nausea and vomiting after breast surgery: efficacy of prophylactic ondansetron and droperidol in a randomized placebo-controlled study. Eur J Anaesthesiol. 2000;17(3):197-203.

15. Conway B. Prevention and management of postoperative nausea and vomiting in adults. Aorn J. 2009;90(3):391-413.

16. Myles PS, Wengritzky R. Simplified postoperative nausea and vomiting impact scale for audit and post-discharge review. Br J Anaesth. 2012;108(3):423-429.

17. Caristi D, Miotto L, Piva M. Pain management and patient satisfaction. In: Gullo A, ed. Anaesthesia, Pain, Intensive Care and Emergency A.P.I.C.E. Springer Milan; 2006:819-830.

18. Chalifoux F, Colin F, St-Pierre P, Godin N, Brulotte V. Low dose intravenous dexamethasone ( $4 \mathrm{mg}$ and $10 \mathrm{mg}$ ) significantly prolongs the analgesic duration of single-shot interscalene block after arthroscopic shoulder surgery: a prospective randomized placebo-controlled study. Can J Anaesth. 2017;64(3):280-289.

19. Boughey JC, Goravanchi F, Parris RN, et al. Improved postoperative pain control using thoracic paravertebral block for breast operations. Breast J. 2009;15(5):483-488. 
20. Moller JF, Nikolajsen L, Rodt SA, Ronning H, Carlsson PS. Thoracic paravertebral block for breast cancer surgery: a randomized double-blind study. Anesth Analg. 2007;105(6):1848-1851.

21. Bhuvaneswari V, Wig J, Mathew PJ, Singh G. Post-operative pain and analgesic requirements after paravertebral block for mastectomy: a randomized controlled trial of different concentrations of bupivacaine and fentanyl. Indian J Anaesth. 2012;56(1):34-39.

22. Boughey JC, Goravanchi F, Parris RN, et al. Prospective randomized trial of paravertebral block for patients undergoing breast cancer surgery. Am J Surg. 2009;198(5):720-725.

23. Abdallah FW, Morgan PJ, Cil T, et al. Ultrasound-guided multilevel paravertebral blocks and total intravenous anesthesia improve the quality of recovery after ambulatory breast tumor resection. Anesthesiology. 2014;120(3):703-713.
24. Goravanchi F, Kee SS, Kowalski AM, Berger JS, French KE. A case series of thoracic paravertebral blocks using a combination of ropivacaine, clonidine, epinephrine, and dexamethasone. J Clin Anesth . 2012;24(8):664-667.

25. Karanicolas PJ, Smith SE, Kanbur B, Davies E, Guyatt GH. The impact of prophylactic dexamethasone on nausea and vomiting after laparoscopic cholecystectomy: a systematic review and meta-analysis. Ann Surg. 2008;248(5):751-762.

26. Callery MP. Preoperative steroids for laparoscopic surgery. Ann Surg. 2003;238(5):661-662.

27. Hargreaves KM, Costello A. Glucocorticoids suppress levels of immunoreactive bradykinin in inflamed tissue as evaluated by microdialysis probes. Clin Pharmacol Ther. 1990;48(2):168-178.

\section{Journal of Pain Research}

\section{Publish your work in this journal}

The Journal of Pain Research is an international, peer reviewed, open access, online journal that welcomes laboratory and clinical findings in the fields of pain research and the prevention and management of pain. Original research, reviews, symposium reports, hypothesis formation and commentaries are all considered for publication

\section{Dovepress}

The manuscript management system is completely online and includes a very quick and fair peer-review system, which is all easy to use. Visit http://www.dovepress.com/testimonials.php to read real quotes from published authors. 